\title{
A Method of Improving SCR for FM-CW Radar
}

\author{
Fumio Nishiyama* and Hideo Murakami*
}

\begin{abstract}
This paper proposes a new method for improving signal-to-clutter ratio (SCR) for frequency modulated continuous wave $(\mathrm{FM}-\mathrm{CW})$ radar. Received signals can be categorized into two types due to either a target or clutter. Target surfaces are generally smoother than clutter surfaces, and thus target signals with different carrier frequencies tend to have stronger correlation than that of clutter. The autocorrelation of linearly FM waveforms from the target and the clutter, when the frequency range is chosen to be large enough, are expected to be significantly different. Based on this statistical difference, the target statistical characteristic is estimated from received signals only, and a matched filter is then designed for improving SCR.
\end{abstract}

Key Words : matched filter, signal-to-clutter ratio, FM-CW radar, automotive radar

\section{Introduction}

Frequency modulated continuous wave (FM-CW) radar transmits a waveform whose frequency changes linearly in time. The $\mathrm{FM}-\mathrm{CW}$ radar can increase transmission energy without increasing peak power by employing continuous waves instead of pulses used in usual radar systems ${ }^{1)}$. This property is particularly advantageous for automotive radars ${ }^{2), 3}$ used for the intelligent transport systems (ITS). The automotive radars, being placed at a position near the ground, inevitably receive clutter from various objects, such as road asphalt, sidewalk lines and objects on the sidewalks.

Clutter is a major factor in causing false alarms in target detection. For a single carrier frequency radar system, suppressing clutter energy is achieved by processing in the time-domain ${ }^{4), 5)}$ or by using the wavelet transform exploiting a difference between power spectra of a target and clutter ${ }^{6}$.

As opposed to the single carrier frequency radar system, stepped FM radar is suggested for suppressing clutter energy and improving range resolution ${ }^{7), 8}$. It uses multiple carrier frequencies with the sufficiently large frequency separation so that clutter signals of different frequencies are regarded as being uncorrelated ${ }^{7,8)}$. In this case, a constant probability of the false alarms is maintained by using constant false alarm rate (CFAR) processing ${ }^{7}$, and

\footnotetext{
* Information and Computer Engineering, Kanazawa Institute of Technology, Ohgigaoka 7-1, Nonoichi, Ishikawa

(Received January 5, 2007)

(Reviced May 11, 2007)
}

the range resolution is improved by using multiple signal classification (MUSIC) method or by taking discrete Fourier transform (DFT) of received signals with different carrier frequencies ${ }^{8)}$. With improving range resolution, the clutter energy is suppressed ${ }^{8)}$. As a different method from these techniques, autocorrelation functions for signals of different carrier frequencies are estimated from received data, and then this estimate is used to design a matched filter for improving signal-toclutter ratio (SCR) under the assumption that clutter signals of different frequencies are completely uncorrelat$\mathrm{ed}^{9)}$.

Easing the above assumption, this paper proposes a method of improving SCR for the linear FM systems under the assumption that the autocorrelation functions for a target and clutter are significantly different. This difference is exploited for estimating the target autocorrelation, and the estimate is then used for improving SCR.

This paper is organized as follows. In Chap. 2, the FM$\mathrm{CW}$ radar system is briefly explained and the received signal model is formulated as a stochastic process with respect to different frequencies. Chapter 3 describes the proposed methods of estimating the target power spectrum and of a procedure for exploiting the estimated power spectrum for increasing SCR. In Chap. 4, the improvement of SCR is analyzed by using a moving average (MA) model for targets. And then the proposed method is evaluated via computer simulation using a specific target model. 


\section{FM-CW radar}

\subsection{Received data}

The FM-CW radar is briefly explained. Fig. 1 shows a block diagram of the $\mathrm{FM}-\mathrm{CW}$ radar using two antennas. The signal generator generates a train of triangular pulses like a saw-tooth shape ${ }^{10)}$, and then the modulator converts the train of pulses into a carrier frequency waveform sweeping its frequency according to the pulse train. Then the waveform is radiated into space through the transmitting antenna.

Echoes reflected from objects, either targets or clutter, are received via the receiving antenna. The received carrier frequency waveform is mixed with the present transmitted carrier frequency waveform to extract a beat waveform. The beat waveform is sampled, and then multiplied by a window function. A matched filter is designed from the window output, and then the received signal norm at the matched filter output is calculated.

Fig. 2(a ) shows a transmitted signal and a received signal for the FM-CW radar employing the saw-tooth modulation $^{11)}$. The carrier frequency linearly varies from $f_{\min }$ to $f_{\max }$, returns to $f_{\min }$, and repeats this cycle as shown in the figure. The time interval for the frequency-change cycle is denoted as $T$.

At the receiver, the mixer together with a low-pass filter, which is not explicitly shown in the figure though, produces the beat waveform ${ }^{10}$. If the beat frequency has a peak at a frequency $f_{p}$, then it has high possibility that a target exists at the distance $c T_{p} / 2$, where $c$ is the speed of light and

$$
T_{p}=\frac{f_{p} T}{f_{\max }-f_{\min }} .
$$

Although a number of peaks may be used for our method, a single peak will be used for a simple explanation. There may or may not exist a target at the distance $c T_{p} / 2$.

The beat waveform is sampled, and then multiplied by a series of windows. Fig. 2 ( b ) shows the series of windows to be multiplied. The duration of each window is the same

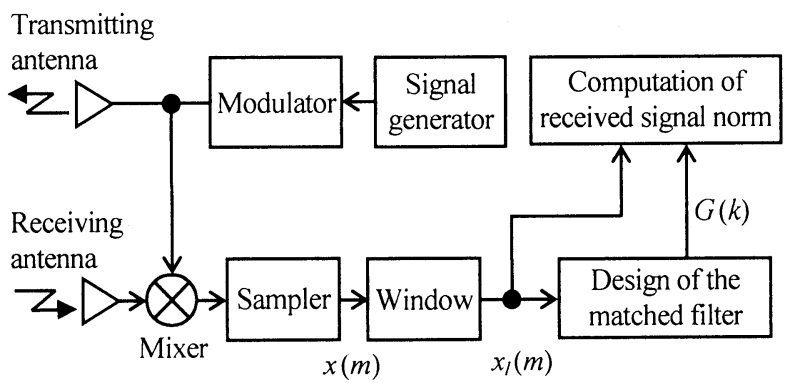

Fig. 1 A block diagram of the FM-CW radar system as the one sweeping time interval $T$, and the start of the first window is delayed by $T_{p}$ as shown in the figure.

The sampler samples the beat waveform with a time interval $T / M$, and produces $M$ samples during one sweeping time $T$. If the samples are denoted as $x(n)$, the output of $l$ th window is written as

$$
x_{l}(m)=x(l M+m), \quad 0 \leq m \leq M-1 .
$$

As $m$ goes from 0 to $M-1$, the carrier frequency changes from $f_{\min }$ to $f_{\max }$. Therefore $m$ corresponds to the carrier frequency

$$
f_{m}=f_{\min }+m \Delta f, \quad 0 \leq m \leq M-1,
$$

where $\Delta f$ is the step of the carrier frequencies given by

$$
\Delta f=\frac{f_{\max }-f_{\min }}{M-1} .
$$

If $\Delta f$ is chosen to be greater than $20 \mathrm{MHz}, x_{l}(m)$ and $x_{l}\left(m^{\prime}\right), m \neq m^{\prime}$, have a weak correlation ${ }^{8)}$. The window output is arranged in the $M$-dimensional vector as

$$
\mathbf{x}_{l}=\left[\begin{array}{llll}
x_{l}(0) & x_{l}(1) & \cdots & x_{l}(M-1)
\end{array}\right] .
$$

Although the index $m$ stands for the frequency $f_{m}, x_{l}(m)$ will be treated as a signal in a manner similar to the usual time-domain representation of signals.

The sample $x_{l}(m)$ follows the statistics of a received target echo or a clutter due to the carrier frequency $f_{m}$ depending on whether there exists a target or not at the distance $c T_{p} / 2$. Let $\mathbf{y}_{T}$ and $\mathbf{y}_{c}$ be stochastic processes corresponding to received signals due to the target and the clutter respectively. We take $L$ of these vectors and the numerical average of the vectors as

$$
\mathbf{y}=\left[\begin{array}{llll}
y(0) & y(1) & \cdots & y(M-1)
\end{array}\right]=\frac{1}{L} \sum_{l=0}^{L-1} \mathbf{x}_{l} .
$$

Since $\mathbf{y}_{T}$ and $\mathbf{y}_{C}$ are stochastic processes, $\mathbf{y}$ can be regard

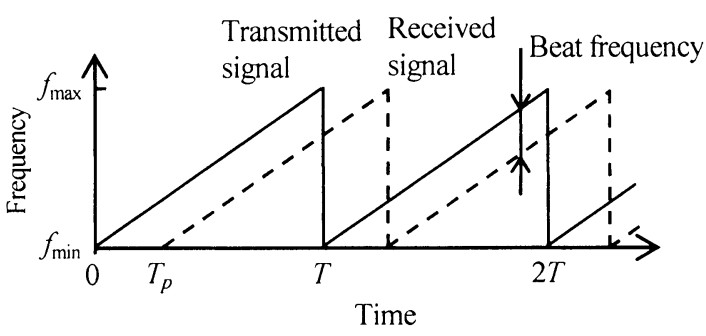

(a) Transmitted signal and received signal

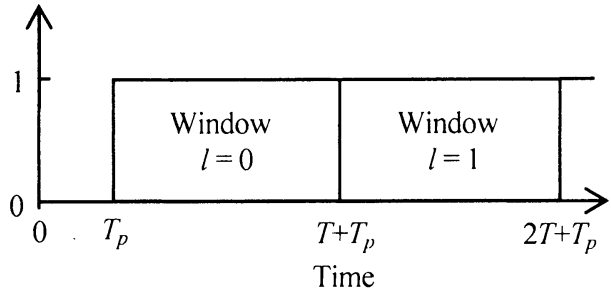

(b) Window

Fig. 2 Transmitted signal, received signal and window function for the $\mathrm{FM}-\mathrm{CW}$ radar system 
ed as a stochastic process. When $L$ is large enough, $\mathbf{y}$ approaches to

$$
\mathbf{y}=P_{T} \mathbf{y}_{T}+P_{C} \mathbf{y}_{C},
$$

where $P_{T}$ and $P_{C}$ are probabilities of occurrence of the target and the clutter. In the following discussion, we assume $L$ is large in order to satisfy ( 7 ).

\section{2 Power spectrum of target signals}

The autocorrelation of $\mathbf{y}$ is defined as

$$
\phi_{Y}(\tau)=E\{y(m) y(m+\tau)\}, \quad-M / 2 \leq \tau \leq M / 2-1,(8)
$$

where $E\{\cdot\}$ denotes the expectation operation ${ }^{12)}$. We need to estimate an autocorrelation of the target process $\mathbf{y}_{T}$ from the observable data $\mathbf{y}$ for designing a filter to improve SCR.

Scattering behavior of traffic targets and ground clutter such as grass and various road surfaces is studied and shown to be influenced by roughness of these surfaces and carrier frequencies ${ }^{7), 8), 13)}$. Statistical analysis for more general types of ground clutter including a corn field and a forest appears in literature ${ }^{14), 15)}$. As a wavelength of carrier changes, the reflection from ground clutter having a rough surface scatters more than that of objects having a smooth surface. Targets such as an automobile have generally smoother surface than ground clutter. Therefore target echoes with different carrier frequencies tend to have stronger correlation than that of clutter ${ }^{16)}$.

Based on the above observations, we assume the followings.

Assumption 1: The two processes $\mathbf{y}_{T}$ and $\mathbf{y}_{C}$ are statically independent with zero-mean.

Assumption 2: The width of the autocorrelation of the target process is much larger than that of clutter one.

By $(7)$ and the assumption 1, the autocorrelation $\phi_{Y}(\tau)$ of received data is obtained as

$$
\phi_{Y}(\tau)=P_{T}^{2} \phi_{T}(\tau)+P_{C}^{2} \phi_{C}(\tau),
$$

where $\phi_{T}(\tau)$ and $\phi_{C}(\tau)$ are the autocorrelation of the processes $\mathbf{y}_{T}$ and $\mathbf{y}_{C}$ respectively. Taking $M$-point DFT of the autocorrelation, the power spectrum of the process $\mathbf{y}$ is given by

$$
\Phi_{Y}(k)=P_{T}^{2} \Phi_{T}(k)+P_{C}^{2} \Phi_{C}(k),
$$

where $\Phi_{T}(k)$ and $\Phi_{C}(k)$ are the target and the clutter power spectrum respectively.

The assumption 2 says that the bandwidth of $\Phi_{T}(k)$ is much narrower than the bandwidth of $\Phi_{C}(k)$. Interpreting this in the equation $(9)$, if $\phi_{T}(\tau)$ is regarded as a signal to be recovered and $\phi_{C}(\tau)$ is noise, then $\phi_{Y}(\tau)$ is the sum of the signal with narrow bandwidth and the noise with broad bandwidth. Therefore, the target autocorrelation function $\phi_{T}(\tau)$ is recovered from $\phi_{Y}(\tau)$ by a low-pass filter in a manner similar to the method of recovering a signal contaminated with a wide band additive noise. Once $\phi_{T}(\tau)$ is recovered, the target power spectrum $\Phi_{T}(k)$ is obtained by taking DFT of $\phi_{T}(\tau)$.

\section{Improving SCR at the receiver}

\section{1 Estimation of the target power spectrum}

In order to proceed with estimating the target power spectrum described at the end of the previous chapter, we need to estimate the autocorrelation $\phi_{Y}(\tau)$ from received signals. So as to do this, we first compute the $M \times M$ matrix $\mathbf{B}$ from the received signals by

$$
\mathbf{B}=\frac{1}{L}\left[\begin{array}{c}
\mathbf{x}_{0} \\
\mathbf{x}_{1} \\
\vdots \\
\mathbf{x}_{L-1}
\end{array}\right]^{*}\left[\begin{array}{c}
\mathbf{x}_{0} \\
\mathbf{x}_{1} \\
\vdots \\
\mathbf{x}_{L-1}
\end{array}\right],
$$

where the superscript ${ }^{*}$ denotes the complex conjugate transpose operation. The $(m, n)$ th entry of this matrix is given by

$$
b_{m, n}=\frac{1}{L} \sum_{l=0}^{L-1} x_{l}^{*}(m) x_{l}(n) .
$$

Evidently the matrix $\mathbf{B}$ approximates the autocorrelation matrix of received signals when $L$ is sufficiently large.

The autocorrelation function is approximated from $\mathbf{B}$ as follows. The $M / 2$-dimensional vector $\mathbf{v}$ is computed according to

$$
\begin{aligned}
\mathbf{v} & =\left[\begin{array}{llll}
v(0) & v(1) & \cdots & v(M / 2-1)
\end{array}\right] \\
& =\frac{2}{M} \sum_{i=0}^{M / 2-1}\left[\begin{array}{llll}
b_{i+M / 4-1, i} & b_{i+M / 4-1, i+1} & \cdots & b_{i+M / 4-1, i+M / 2}
\end{array}\right] .
\end{aligned}
$$

Then the approximation of the autocorrelation function of received signals is obtained as

$$
\hat{\phi}_{Y}(\tau)=v(M / 4+\tau-1) .
$$

The target autocorrelation $\phi_{T}(\tau)$ may be estimated from the above estimate by the method explained at the end of the previous chapter. In particular when the clutter process is white, by (9) we have

$$
\phi_{Y}(\tau)=P_{T}^{2} \phi_{T}(\tau), \quad \tau \neq 0 .
$$

This means that the shape of $\phi_{T}(\tau), \tau \neq 0$ is identical to the shape of $\phi_{Y}(\tau)$ apart from the constant multiplication by $P_{T}^{2}$. The value of $\phi_{T}(\tau)$ at $\tau=0$ can be estimated from its known neighbors in $\phi_{Y}(\tau)$ by a standard interpolation method. Then target power spectrum $\Phi_{T}(k)$ is estimated by taking DFT of the estimate of $\phi_{T}(\tau)$.

The above-mentioned method works regardless of the location of a target, as seen from the fact that the matrix B computed by (11) is independent of exchange of received signals. For example, the exchange of $\mathbf{x}_{0}$ and $\mathbf{x}_{1}$ results in the same matrix. However, in cases when there is no target or when the number of target signals is not 
enough within $L$ windows, the method fails to estimate the target autocorrelation. Therefore, the target power spectrum can not be accurately estimated. This dependence of the estimate on the number of target signals is analyzed in Chap. 4.

\section{2 Computation of the received signal norm}

Once the power spectrum of the target is estimated, it is now ready to process the received signals to improve SCR using the estimated target power spectrum $\bar{\Phi}_{T}(k)$. We first prepare the matched filter $G(k)$ given as

$$
G(k)=\sqrt{\frac{M}{\sum_{m=0}^{M-1}\left|\widehat{\Phi}_{T}(m)\right|}} \sqrt{\left|\widehat{\Phi}_{T}(k)\right| .}
$$

The matched filter $G(k)$ is normalized so that the sum of $|G(k)|^{2}, 0 \leq k \leq M-1$, becomes $M$.

Using the matched filter $G(k)$, we perform the steps described in Fig. 3. The $M$-point DFT $X_{l}(k), 0 \leq k \leq M-1$, of $x_{l}(m), 0 \leq m \leq M-1$, is computed, and then each DFT value is multiplied by the matched filter to obtain its output $Z_{l}(k)$ as

$$
Z_{l}(k)=G(k) X_{l}(k), \quad 0 \leq k \leq M-1 .
$$

Finally, the norm of this matched filter output $Z_{l}(k)$ is calculated by

$$
P_{l}=\frac{1}{M} \sum_{k=0}^{M-1}\left|Z_{l}(k)\right|^{2}
$$

This norm $P_{l}$ takes a large value when there is a target at the time of window. $l$.

\section{Evaluation of the method}

\section{1 Improvement of SCR}

The received signal $x_{l}(m)$ becomes either the random variable $y_{T}(m)$ or $y_{C}(m)$ depending on whether there is a target or clutter at the time of window $l$. Based on this observation, we assign, in the place of $P_{l}$, two random variables

$$
Q_{i}=\frac{1}{M} \sum_{k=0}^{M-1}\left|G(k) Y_{i}(k)\right|^{2}, \quad i=T, C,
$$

where $Y_{T}(k)$ and $Y_{C}(k)$ are the $M$-point DFTs of $y_{T}(m)$ and $y_{c}(m)$ respectively. Therefore, $Q_{T}$ and $Q_{C}$ are the norm of the matched filter output when a target or clutter is received. Evidently, phase components of the matched filter do not affect to the computation of the norm.

From the relation of $\Phi_{i}(k)=(1 / M) E\left[\left|Y_{i}(k)\right|^{2}\right], i=T, C$, the mean of the norm is given by

$$
E\left\{Q_{i}\right\}=\sum_{k=0}^{M-1}|G(k)|^{2} \Phi_{i}(k), \quad i=T, C .
$$

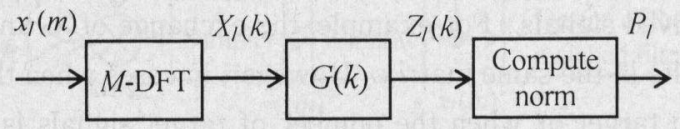

Fig. 3 Steps for computing the received signal norm
Therefore, SCR at the output of the matched filer is given as

$$
S C R=\frac{E\left\{Q_{T}\right\}}{E\left\{Q_{C}\right\}}=\frac{\sum_{k=0}^{M-1}|G(k)|^{2} \Phi_{T}(k)}{\sum_{k=0}^{M-1}|G(k)|^{2} \Phi_{C}(k)}
$$

This equation means that SCR is equal to one, and it can not be improved by using the matched filter when the power spectra of the target and the clutter are the same.

Fig. 4 demonstrates how SCR is improved by the proposed method assuming that the clutter power spectrum is flat and the target power spectrum is narrow band. The frequency response of the matched filter is shown in Fig. 4(a) when the target power spectrum is perfectly estimated. The power spectrum of the target is then given as the absolute value of the response. Fig. 4( b ) shows the power spectra of the target and the clutter at the input of the matched filter. The power spectra at the output of the matched filter are shown in Fig. 4(c).

At the output of the matched filter, the target power barely attenuates by the matched filter, whereas the clutter power considerably decreases by the stop-band of the matched filter. Therefore, the method improves SCR provided that the target power spectrum is narrower than the clutter power spectrum and the target power spectrum is correctly estimated.

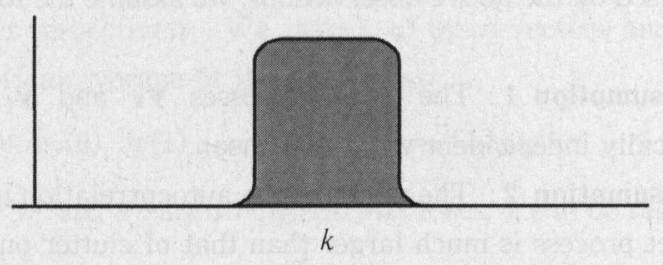

(a) Frequency response of the matched filter

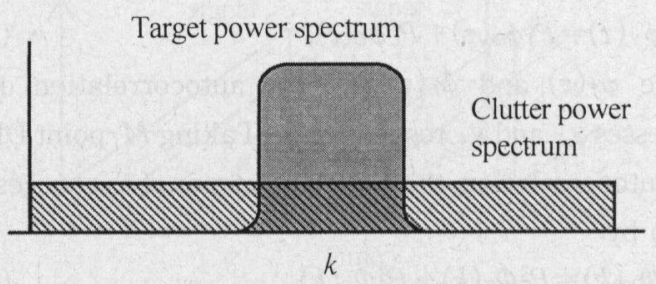

(b) Power spectra of target data and clutter data at the input of the matched filter

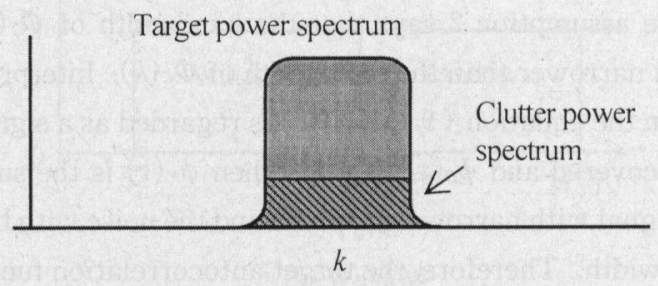

(c) Power spectra at the output of the matched filter

Fig. 4 Mechanism of improving SCR by the proposed method 
Example: Auto-regressive (AR) and auto-regressive moving-average (ARMA) models are employed for targets in a single carrier radar system ${ }^{17}$. However it seems that there is no standard signal model for a multiple carrier frequency radar system. In order to get a general idea of a statistical model for targets with respect to different carrier frequencies, we have measured an autocorrelation by a numerical averaging method using a $60 \mathrm{GHz}$ band FM-CW radar system in an anechoic chamber. Table 1 shows specifications of the radar system for measuring target signals. The autocorrelation coefficients are shown as the solid line in Fig. $\mathbf{5}$ using a flat aluminum board with its surface facing the antenna as a target.

The method works as long as the assumption 1 and 2 are satisfied, and does not require a specific statistical model for a target. However, we use the MA model simply because it provides a relatively simple autocorrelation, and enables us to compare the autocorrelation obtained by the analysis and autocorrelations obtained by simulations. The MA model is given by

$$
y_{T}(n)=\sum_{u=0}^{K-1} h(u) w(n-u),
$$

where $h(u), 0 \leq u \leq K-1$, denotes MA parameters ${ }^{18)}$. $w(n)$ is a process of zero-mean white Gaussian and $K$ is the order of the model. The power spectrum of $y_{T}(n)$ is given by

Table 1 Specifications of the FM-CW radar system

\begin{tabular}{l}
\hline \hline Center frequency : $60 \mathrm{GHz}$ \\
Frequency bandwidth $: 700 \mathrm{MHz}$ \\
Transmitting power : $10 \mathrm{dBm}$ \\
\hline \hline
\end{tabular}

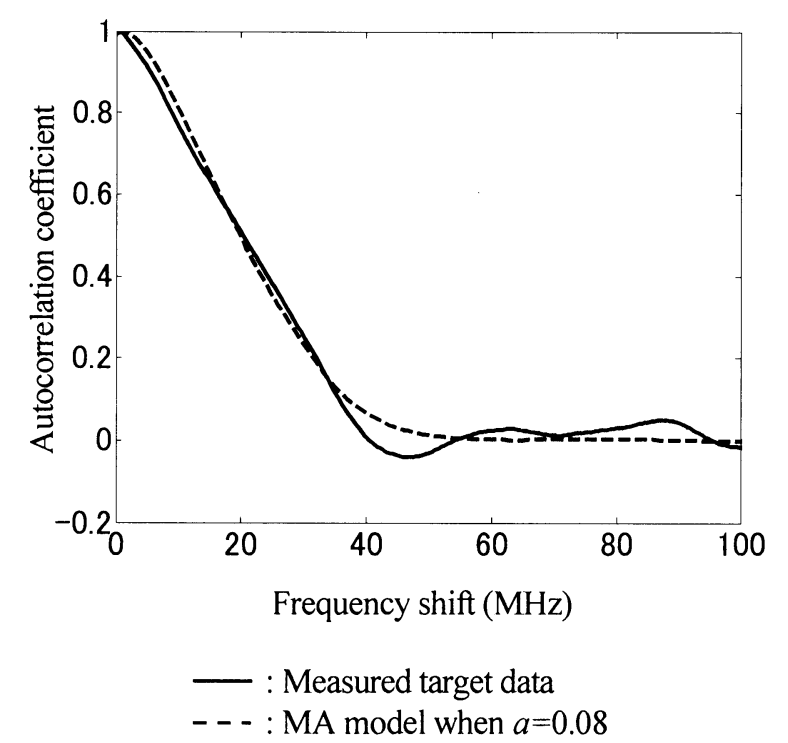

Fig. 5 Autocorrelation coefficients of signals from an aluminum flat board and a MA model

$$
\Phi_{T}(k)=\sigma_{w}^{2}|H(k)|^{2},
$$

where $\sigma_{w}^{2}$ is the variance of $w(n)$ and $H(k)$ is DFT of $h(n)^{18)}$.

As the MA parameters, we consider the form given by

$$
h(n)=e^{-a|n|}
$$

where $a$ is a positive constant. The constant $a$ controls the shape of the autocorrelation. The autocorrelation of the MA model is shown as the dotted line in Fig. 5, in which the constant $a$ is deliberately chosen to suit to the case of the aluminum board target.

DFT of $h(n)$ given by (24) is obtained as

$$
\begin{aligned}
H(k) & =\sum_{n=0}^{N-1} h(n) e^{-2 \pi n k / N} \\
& =\frac{\left(1-e^{-2 a}\right)\left(1-(-1)^{k} e^{-a N / 2}\right)}{1-2 e^{-a} \cos (2 \pi k / N)+e^{-2 a}} .
\end{aligned}
$$

This DFT is shown in Fig. 6. As the constant $a$ increases, the bandwidth of $H(k)$ increases and thus the bandwidth of the target power spectrum increases.

For evaluating the method, it is necessary to compare SCRs in the cases of which the matched filter is used and not used. Substituting $G(k)=1$ into (21), SCR is given by

$$
S C R_{0}=\frac{\sum_{k=0}^{M-1} \Phi_{T}(k)}{\sum_{k=0}^{M-1} \Phi_{C}(k)}
$$

when the matched filter is not used. We analyze the performance of the method by the improvement ratio of SCR of (21) to the $S C R_{0}$ defined by

$$
R_{S C R}=\frac{S C R}{S C R_{0}} \text {. }
$$

The ratio $R_{S C R}$ is shown as a function of $a$ in Fig. 7 assuming that the clutter is white, and that the MA model whose parameters given by (24) is used for targets.

The improvement ratio is compared with three conventional methods: the integration processing method ${ }^{11)}$, the discrete wavelet transform method $^{6), 19)}$ and the DFT

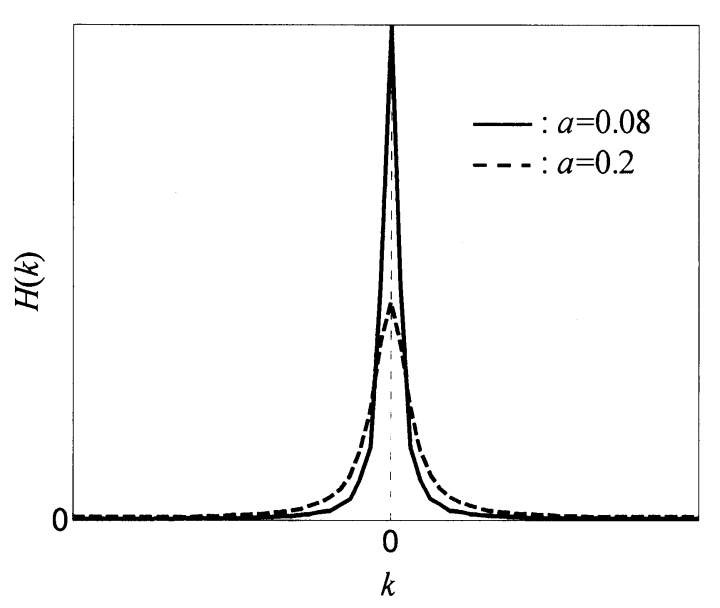

Fig. 6 DFT $H(k)$ of $h(n)$ given by (24) 


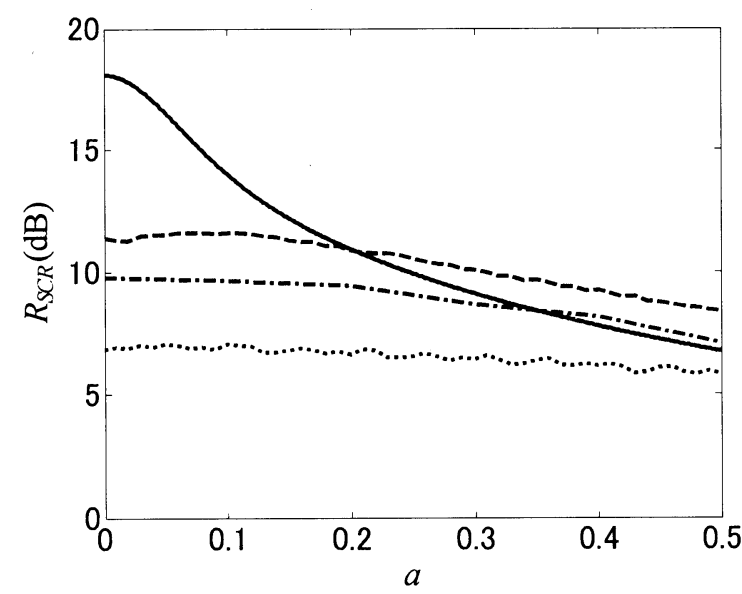

- : Proposed method

- - - : Discrete wavelet transform

- - : DFT

....... : Integration processing

Fig. $7 R_{S C R}$ as a function of the constant $a$

method $^{8)}$. For the integration processing, the improvement ratio of SCR at 7 windows is computed. For the discrete wavelet transform method, the scaling function of 12 orders of the Daubechies wavelet is employed. For the DFT method, the improvement ratio by taking DFT using 10 windows is computed. The improvement ratios of these conventional methods are also exhibited in Fig. 7. The ratio $R_{S C R}$ decreases as $a$ increases, which in turn implies the increase of the target spectrum bandwidth. When the constant $a$ is less than 0.2 , the performance of the proposed method exceeds the other methods, assuming that the autocorrelation is perfectly estimated.

\section{2 Simulation}

When the number of target signals within $L$ windows is large enough, the estimate of the target power spectrum becomes accurate. However the performance accordingly degrades when the number is small. Simulations are performed to see how many target signals are needed to acquire a desirable performance.

Simulation parameters used in the computer simulation are listed in Table 2. The MA models in Example are used for the present simulations as well. The target signals are produced by the convolution of $h(n)$ and the random number with zero-mean white Gaussian. The clutter signals are created as the random number with zero-mean white Gaussian. Fig. 8 shows the ratio $R_{S C R}$ as a function of the number of target signals in $L$ windows. As seen from the figure, SCR improves as the number of target vectors increases, and saturates after the certain number of target vectors. The ratio $R_{S C R}$ improves by decreasing the constant $a$ as expected from Fig. 7 .
Table 2 Simulation parameters

The constant $a$ of the MA parameter : 0.08 and 0.2

Size of received signals : $L \times M=512 \times 64$

The number of target vectors : $0-10$

The order of the matched filter : 64

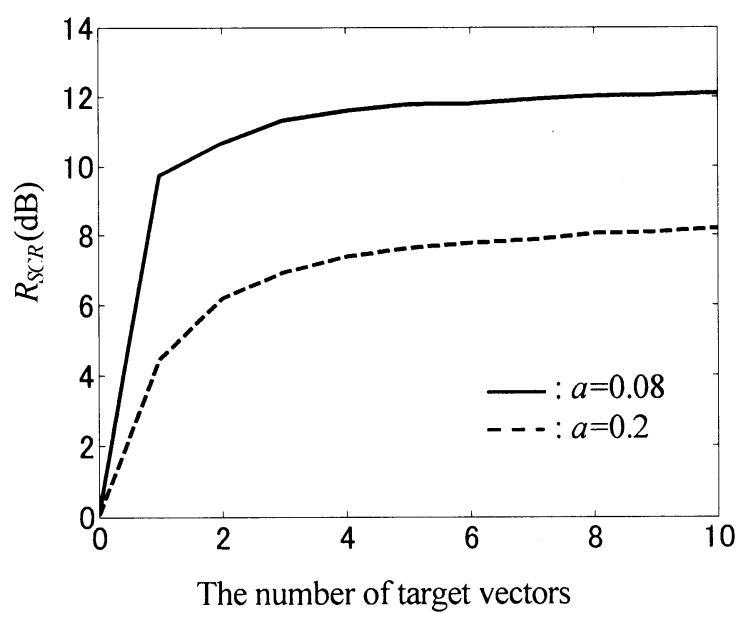

Fig. $8 R_{S C R}$ as a function of the number of target signals

When the target number is zero, the ratio is one because such SCR is equal to the case when the matched filter is not used at all. When the number of target signals is not large enough, the ratio does not reach its value given by (27) because the target power spectrum is not accurately estimated. If this is the case, the problem is eased by increasing the number $L$ of the windows.

\section{Conclusions}

This paper has introduced the method of improving SCR for the $\mathrm{FM}-\mathrm{CW}$ radar under the assumption that received signals from a target with different carrier frequencies have a stronger correlation than the received signals from clutter. The method consists of two phases.

The first phase estimates the autocorrelation of the received signals with different carrier frequencies by accumulating enough number of received signals. And then the target autocorrelation is extracted by exploiting the difference between the statistics of the target and the clutter. The estimate of the target power spectrum is obtained by taking DFT of the target autocorrelation.

At the second phase, the matched filter is designed from the knowledge of the target power spectrum. Then the steps described in Fig. 3 are performed to obtain the norm of the received signals given by (18).

The performance of the proposed method was analyzed in terms of SCR using the MA model for targets. The simulations verified that the proposed method is useful for 
improving SCR for the FM-CW radar systems.

\section{References}

1) M. I. Skolnik : Introduction to radar systems, third edition, 195/196, McGraw-Hill Book Company (2001)

2) T. Horimatsu and M. Hitotsuya: Millimeter-wave radar in practical use, J. IEICE, 87-9, 756/759 (2004)

3) H. Kondoh, H. Nagaishi, H. Shinoda, N. Kurita, T. Nagasaku and K. Takano: Millimeter-wave automotive radar sensors for ITS applications, IEICE Trans. Electron. (Japanese Edition), J88-C-8, 613/620 (2005)

4) S. Kouya and S. Miwa: A method of SCR improvement with a two-dimensional weighted moving average filter, IEICE Trans. Commun. (Japanese Edition), J83-B-3, 361/ 369 (2000)

5) M. Shimizu, S. Kouya, S. Miwa and N. Kaku : Radar SCR improvement using a contraction method, IEICE Trans. Commun. (Japanese Edition), J83-B-7, 1058/1066 (2000)

6) A. Saitou, S. Kouya, S. Miwa and N. Kaku: Radar SCR improvement using a wavelet transform, IEICE Trans. Commun. (Japanese Edition), J84-B-5, 923/931 (2001)

7) H. Yamaguchi, A. Kajiwara, S. Hayashi, R. Saiga, F. Nishiyama and K. Serizawa: Target detection in ground clutter with millimeter-wave stepped frequency radar, IEICE Technical Report, SANE 2001-16, 31/36 (2001)

8) A. Kajiwara and H. Yamaguchi : Clutter suppression characteristics of stepped-FM radar with MUSIC algorithm, IEICE Trans. Commun. (Japanese Edition), J84-B-10, $1848 / 1856$ (2001)

9) F. Nishiyama and H. Murakami: Blind matched filter method for FM-CW radar, Proceedings of IASTED SIP Conference, SIP 2006, proceeding 534, 534-083 (2006)

10) S. Tanaka and N. Harada: Remote sensing system for localization of plural objects using a FM-CW-type electromagnetic wave (radar), T. SICE, 40-12, 1199/1204 (2004)

11) M. I. Skolnik: Radar handbook, second edition, 14.21/ 14.28, 2.17, McGraw-Hill Book Company (1990)

12) A. Papoulis and S. U. Pillai: Probability, random variables, and stochastic processes, fourth edition, 420, McGraw-Hill Book company (2002)

13) E. S. Li and K. Sarabandi : Low grazing incidence millimeter-wave scattering models and measurements for various road surfaces, IEEE Trans. Antennas Propagat., 47-5, 851/861 (1999)

14) F. T. Ulaby and M. C. Dobson: Handbook of radar scattering statistics for terrain, 70/78, Artech House (1989)
15) F. T. Ulaby, T. F. Haddock and R. T. Austin: Fluctuation statistics of millimeter-wave scattering from distributed targets, IEEE Trans. Geosci. Remote Sensing, 26-3, 268/ 281 (1988)

16) K. Serizawa and T. Yamaoka : Scattering characteristics in $\mathrm{W}$-band millimeter wave, Proc. IEICE Gen. Conf. '04, Communication 1, 299 (2004)

17) P. Thomas and S. Haykin: Stochastic modeling of radar returns, IEE Proc., Vol. 133, Pt. F, No. 5, 476/481 (1986)

18) W. A. Fuller: Introduction to statistical time series, second edition, 21/26, John Wiley \& Sons, Inc. (1996)

19) H. Nakano, S. Yamamoto and Y. Yoshida: Signal processing and image processing by using wavelet transform, 74/ 94, Kyoritsu Publication (1999)

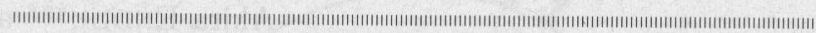
Fumio Nishiyama (Student member)

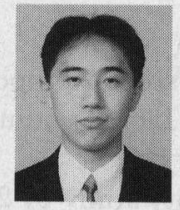

He received the B.E. degree in electrical engineering from Tokyo University of Science, Tokyo, in 1995, and the M.E. degree in information engineering from Kanazawa Institute of Technology, Kanazawa, in 2005. He has been in Technical Research and Development Institute, Ministry of Defense, since 1990. He is also currently with Kanazawa Institute of Technology as a doctorate student. His research interests include digital signal processing and millimeter-wave radar systems.

\section{Hideo Murakayi}

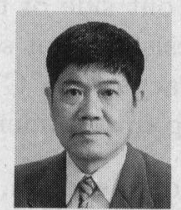

He received the B.S. degree in electrical engineering from Kanazawa University, Kanazawa, in 1968, and the M.S. and Ph.D. degrees in electrical engineering from the University of Southern California, Los Angeles, U.S.A., in 1973 and 1977, respectively. From 1968-1970, he was employed at Mitsubishi Electric Corporation, Hyogo. Since 1977, he has been with Kanazawa Institute of Technology, Kanazawa, where he was an Associate Professor, and is currently a Professor there. His research interests include digital signal processing and coding theory. 